\title{
Azobenzene-functionalized alkanethiols in self-assembled monolayers on gold
}

\author{
Roland Schmidt • Erik McNellis • Wolfgang Freyer • \\ Daniel Brete - Tanja Gießel • Cornelius Gahl • \\ Karsten Reuter · Martin Weinelt
}

Received: 18 February 2008 / Accepted: 9 July 2008 / Published online: 9 August 2008

(C) Springer-Verlag 2008

\begin{abstract}
Self-assembled monolayers (SAMs) of 4-trifluoromethyl-azobenzene-4'-methyleneoxy-alkanethiols $\quad\left(\mathrm{CF}_{3}-\right.$ $\mathrm{C}_{6} \mathrm{H}_{4}-\mathrm{N}=\mathrm{N}-\mathrm{C}_{6} \mathrm{H}_{4}-\mathrm{O}-\left(\mathrm{CH}_{2}\right)_{n}-\mathrm{SH}$ on (111)-oriented polycrystalline gold films on mica were examined by $\mathrm{X}$-ray photoelectron spectroscopy (XPS) and X-ray absorption spectroscopy (XAS). The spectra are analyzed with the help of density-functional-theory calculations of the isolated molecule. Only one doublet is detected in the sulphur $2 p$ spectra of the investigated SAMs, consistent with a thiolate bond of the molecule to the gold surface. The C $1 s$ XP spectra and the corresponding XAS $\pi^{*}$ resonance exhibit a rich structure which is assigned to the carbon atoms in the different chemical surroundings. Comparing XPS binding energies of the azobenzene moiety and calculated initial-state shifts reveals comparable screening of all $\mathrm{C} 1 \mathrm{~s}$ core holes. While the carbon $1 s$ XPS binding energy lies below the $\pi^{*}$-resonance excitation-energy, the reversed order is found comparing core ionization and neutral core excitation of the nitrogen $1 s$ core-hole of the azo group. This surprising difference in core-hole binding energies is interpreted
\end{abstract}

R. Schmidt · W. Freyer · D. Brete · T. Gießel · C. Gahl •

M. Weinelt ( $\varangle)$

Max-Born-Institut, Max-Born-Str. 2A, 12489 Berlin, Germany

e-mail: weinelt@mbi-berlin.de

E. McNellis · K. Reuter

Fritz-Haber-Institut der Max-Planck-Gesellschaft,

Faradayweg 4-6, 14195 Berlin, Germany

M. Weinelt

Fachbereich Physik, Freie Universität Berlin, Arnimallee 14,

14195 Berlin, Germany

T. Gießel

BESTEC GmbH, Carl-Scheele Str. 14, 12489 Berlin, Germany as site-dependent polarization screening and charge transfer among the densely packed aromatic moieties. We propose that a quenching of the optical excitation within the molecular layer is thus one major reason for the low trans to cis photo-isomerization rate of azobenzene in aromaticaliphatic SAMs.

PACS $82.80 . \mathrm{Pv} \cdot 64.75 . \mathrm{Yz} \cdot 68.43 .-\mathrm{h}$

\section{Introduction}

Molecular motion induced by optical excitation provides new prospects for the functionalization of surfaces on the nanoscale. Azobenzene-based photo-switches have become widely explored prototype systems in this respect. The bare azobenzene molecule has two isomers, the thermally stable trans form and the metastable cis form. In solution optical excitation of the $\pi-\pi^{*}$ and $n-\pi^{*}$ transitions reversibly switches between these conformers. The (sub)picosecond time constant of the isomerization reaction in solution yields a quantum efficiency close to unity [1,2], and switching is possible even with a photodiode. In contrast, no successful reversible switching by optical excitation has been achieved to date when the bare azobenzene molecule is adsorbed at a metal surface. This is most likely due to ultrafast charge transfer into the substrate which quenches the optical excitation. Even for a minimized coupling to the substrate as in the case of tert-butyl functionalized azobenzene on $\mathrm{Au}(111)$, the quantum yield decreases by about four orders of magnitude, and laser light is needed to reasonably activate the isomerization reaction $[3,4]$. The latter seems to be mediated by charge transfer from optically excited hot carriers in the substrate to the $\pi^{*}$-LUMO (Lowest Unoccupied Molecular Orbital). As substrate carriers are excited at all wavelengths, 


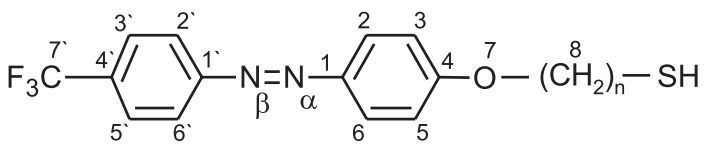

Fig. 1 TF-Azn molecule, where $n$ denotes the length of the alkanethiol chain in form of the number of methylene groups

the optical control over back or forth switching is lost. For application purposes, it thus seems necessary to further decouple the switch from the surface by even larger molecular ligands. On the other hand, bulky spacers usually cause disorder in the molecular layer and prevent preferential orientation of the switch upon adsorption. This reduces the number of potential applications, since concepts for, e.g., switchable surface properties or optical data storage are usually based on aligning molecular tailgroups and dipole moments.

Promising candidates to overcome this problem are selfassembled monolayers (SAMs) [5]. Alkanethiols are known to form SAMs on gold surfaces which are easily prepared by immersion. Due to the specific chemical bond between the thiol headgroup and the gold surface, as well as the lateral interactions among the aliphatic chains, the resulting self-assembled layers are much better ordered than those formed of purely aromatic thiols [6]. Coupling aromatic compounds to aliphatic chains offers therefore a viable strategy to produce films of better structural quality [7], and especially biphenyl-based thiols BP $n$ with different aliphatic chain lengths $\left(-\mathrm{CH}_{2}-\right)_{n}(n=1,2, \ldots, 6)$ have been extensively studied in recent years. In this work, we also follow this approach and use alkanethiols to decouple the azobenzene photo-switch from a gold surface. Figure 1 shows a constitutional formula of the molecule (TF-Azn), in which the azobenzene chromophore is attached via an oxygen atom in the 4 position of the lower phenyl ring to an alkane chain of varying length $n$. The trifluoromethyl-group (TF) in the $4^{\prime}$ position of the second phenyl ring is furthermore used as a marker for core-level spectroscopy. Specifically, high-resolution X-ray photoemission spectroscopy (XPS) and X-ray absorption spectroscopy (XAS) of the carbon and nitrogen near edge X-ray absorption fine structure was employed to study the electronic structure of these molecules in SAMs on gold, analyzing the experimental results with the help of density-functional theory (DFT) calculations on the free molecule.

\section{Experiment}

\subsection{Chemicals and sample preparation}

We synthesized azobenzene-alkanethiols with different aliphatic spacer chain-length $n=3,6$, and 10 between the thiol headgroup and the azobenzene chromophore

$\mathrm{CF}_{3}-\mathrm{C}_{6} \mathrm{H}_{4}-\mathrm{N}=\mathrm{N}-\mathrm{C}_{6} \mathrm{H}_{4}-\mathrm{O}-\left(\mathrm{CH}_{2}\right)_{n}-\mathrm{SH}$.
The unsymmetrical azobenzenethiols containing a trifluoromethyl tailgroup (TF-Azn) were prepared in a three-step process. The starting material used for the synthesis was 4-((4'-trifluoromethyl) azo)phenol. This compound was obtained by coupling the diazonium salt of 4-trifluoromethyl aniline with phenol. 4-((4'-Trifluoromethyl)azo)phenol was then converted to the corresponding $n$ - $\left(4-\left(\left(4^{\prime}-\right.\right.\right.$ trifluoromethylphenyl)azo) phenoxy)alkane-1-bromides $(n=3,6,10)$ by reaction with the $\alpha, \omega$-dibromoalkanes. Thiol derivatization of the $\omega$-bromo group was carried out via the isothiuronium salt.

The azobenzene thiols were chromatographed on silica gel. The purity of the compounds was checked by liquid chromatography (thin layer and HPLC) analysis. The yield of pure azobenzene thiols was 10-30\%. All Azn compounds were characterized by UV/vis-, ${ }^{1} H$ NMR spectroscopy, and mass spectrometry.

As substrates we used commercially available $300 \mathrm{~nm}$ thick gold films evaporated on mica (Georg Albert PVDBeschichtungen). The samples are flame-annealed and were stored in argon atmosphere until further use. Scanning tunneling microscopy (STM) of the as-delivered samples reveal a high crystalline quality with large (111)-oriented terraces of 50-100 nm width, as also confirmed in the recent STM investigation by Kowalczyk [8]. Some samples were flame annealed a second time directly before preparing the SAMs, without observing significant variations in the obtained XPS and XAS data. SAM preparation followed the procedures described in [9]. TF-Az6 and TF-Az10 monolayers were prepared immersing the gold substrate into dilute ethanolic solution $\left(10^{-4} \mathrm{~mol} / \mathrm{l}\right)$ of the respective thiol for 24 hours at room temperature. After removal from solution, the samples were thoroughly rinsed with pure ethanol. The samples were transported in pure ethanol, dried in a stream of argon gas, clamped onto a sample holder, and transferred into vacuum $\left(\leq 10^{-7}\right.$ mbar) within 30 minutes after immersion. All steps were processed under ambient conditions, but using artificial lighting at $560 \mathrm{~nm}$.

\subsection{X-ray spectroscopy}

A two-chamber ultrahigh-vacuum system with a base pressure of $2 \times 10^{-10}$ mbar was used for the XPS and XAS investigations. A fast two-stage load-lock and sample storage allowed us to exchange samples within a few minutes. The actual experiments were performed at the undulator beamlines U41 PGM-1 and UE112 PGM-1 of the Berlin synchrotron facility BESSY 2 [10] using a hemispherical electron analyzer (Omicron EA125). The energy resolution of the $\mathrm{S} 2 p$ XPS measurements was $\Delta R=0.2 \mathrm{eV}$, as established by the width of the Fermi level of the gold substrate. All measurements were performed in grazing incidence with an angle of $\alpha=15^{\circ}$ between $\mathrm{X}$-ray beam and surface plane. 
All spectra are referenced to the binding energy of the $\mathrm{Au}$ $4 f_{7 / 2}$ core level of $83.95 \mathrm{eV}$ [11]. The XPS signals are normalized to the X-ray beam current and decomposed via a least squares fit into Voigt profiles and a Shirley background [12]. The XA spectra were recorded at the $\mathrm{C} 1 s$ and $\mathrm{N} 1 s$ absorption edges in Auger detection-mode sampling the KVVtransition in an energy range of $\pm 5 \mathrm{eV}$ around the peak maxima at 259 and $377 \mathrm{eV}$, respectively. Spectra were also normalized to the photon flux recording the throughput characteristics of the beamline by a photodiode. The photon energy scale is calibrated using the Au $4 f$ line and the Au Fermi edge in combination with the work function of the analyzer. This procedure yields comparable photon-energy scales for both beamlines to within $0.1 \mathrm{eV}$.

\section{Theory}

In order to support the experimental data analysis, a restricted set of density-functional theory (DFT) and timedependent DFT (TD-DFT) calculations was performed using the Gaussian suite of programs [13] and the B3LYP hybrid density functional [14] to treat electronic exchange and correlation. Excitation energies of low lying resonances in the UV spectrum were computed within the response theory formulation of TD-DFT [15], using the B3LYP functional in the adiabatic approximation [16]. Neglecting the effect of the underlying substrate and the surrounding molecules in the SAM, the calculations focused on a single azobenzene unit, functionalized in the opposing phenyl para positions with $\mathrm{CF}_{3}$ group and an oxygen atom bound to a six unit alkanethiol chain as illustrated in Fig. 1. Geometry optimization and excitation energy calculations were carried out using the all-electron def-TZVP basis set [17]. The interpretation of the experimental XP and XA spectra is qualitatively supported by calculated isodensity contours of the energetically low-lying Kohn-Sham orbitals. In order to obtain a more reliable representation of the virtual orbitals, the corresponding computations at the optimized ground-state geometry were based on the more diffuse IGLO-III basis set [18]. Rather than explicitly accounting for screening effects in the excited state, the dominant experimental peaks are then roughly assigned to the virtual orbitals by merely aligning the energetic position of the computed and measured LUMO. In this sense, we also define initial-state "chemical" core level shifts of carbon species in different binding environments as the difference of the $\mathrm{C} 1 s$ Kohn-Sham eigenvalues in the respective cases [19].

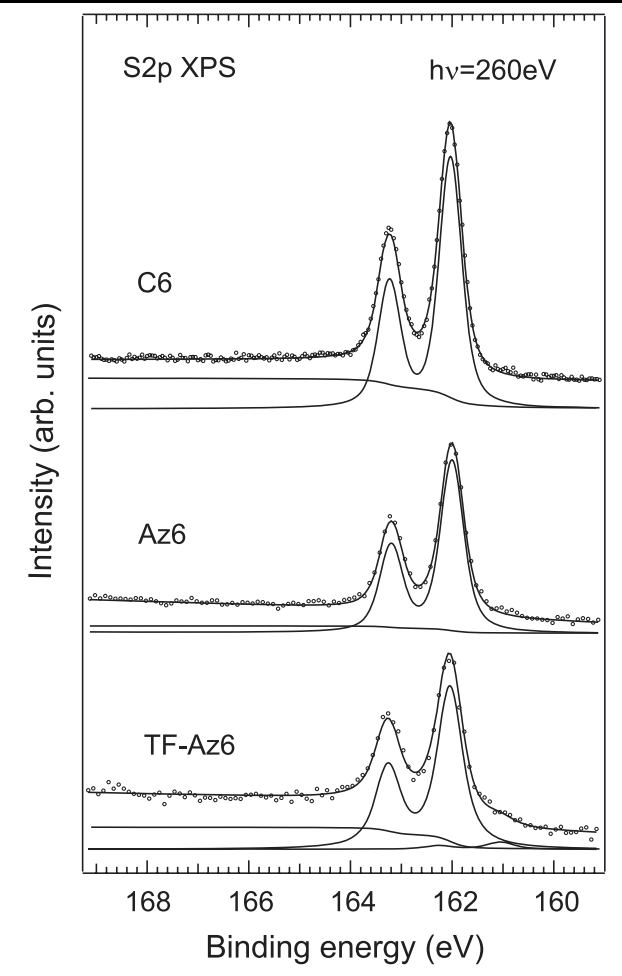

Fig. 2 XP spectra of the S $2 p$ core level of C6, Az6, and TF-Az6 on gold recorded at a photon energy of $260 \mathrm{eV}$ with a resolution of $\Delta R \leq 0.2 \mathrm{eV}$. The spectra are decomposed into least square fits of Voigt profiles and a Shirley background [12]

\section{Results and discussion}

4.1 Core-level binding energies of the azobenzene-based thiols Az6 and TF-Az6

High-resolution XP spectra of the S $2 p$ core level were recorded for SAMs formed from bare alkanethiols, from azobenzene-alkanethiols, and when the molecules were additionally functionalized with the TF marker group (TFAzn). Figure 2 shows the corresponding spectra for alkane chains of length of $n=6$. Binding energies of the decomposed components are additionally listed in Table 1 . The sulphur XPS data are dominated by the $2 p$ doublet with an intensity ratio of two $\left(2 p_{3 / 2} v s .2 p_{1 / 2}\right)$. The binding energies of $162.0 \mathrm{eV}\left(2 p_{3 / 2}\right)$ for Az6, C6, and TF-Az6 are generally assigned to the thiolate species [20]. The latter value was also found in a recent study of biphenyl-based thiols BP $n$ with even chain length $(n=4,6)$ [21]. Heister et al. report on a small odd-even effect for BP3 and BP4 with binding energies of 159.94 and $159.90 \mathrm{eV}$, respectively [20]. The deconvolution of the TF-Az6 compound suggests a small contribution at a binding energy of $161.0 \mathrm{eV}\left(\mathrm{S} 2 p_{3 / 2}\right)$. The latter is attributed either to atomic sulphur or intact molecules bound at distinctly different adsorption sites [20, 2224]. Such a doublet has also been observed for BP4 and BP6, 
Table 1 XPS data for S $2 p_{3 / 2}, \mathrm{C} 1 s$, and $\mathrm{N} 1 s$ for C6, Az6, and TF-Az6 films on Au(111) on mica, respectively. Experimental binding energies in $\mathrm{eV}$ are evaluated with respect to the Au $4 f_{7 / 2}$ core level at $83.95 \mathrm{eV}$ [11]. For HR-XPS measurements with a resolution $\Delta R \leq 0.2 \mathrm{eV}$, the FWHM in $\mathrm{eV}$ is added in parentheses. Based on the alignment with the $\mathrm{Au} 4 f_{7 / 2}$ peak, we estimate an error of $0.1 \mathrm{eV}$ for the individual lines $\mathrm{C}-\mathrm{C}, \mathrm{C}=\mathrm{C}$, $\mathrm{CF}_{2}$, and $\mathrm{CF}_{3}$. All other values follow from a least squares fit and accordingly are model-dependent

\begin{tabular}{|c|c|c|c|c|c|c|c|c|}
\hline C6 & \multicolumn{8}{|c|}{$\mathrm{CH}_{3}-\left(\mathrm{CH}_{2}\right)_{5}-\mathrm{S}-\mathrm{Au}$} \\
\hline $\mathrm{S} 2 p_{3 / 2}$ & $\mathrm{C}-\mathrm{C}$ & \multicolumn{7}{|l|}{$\mathrm{C}-\mathrm{S}$} \\
\hline $162.0(0.52)$ & 284.6 & \multicolumn{7}{|l|}{285.4} \\
\hline Az6 & \multicolumn{8}{|c|}{$\mathrm{C}_{6} \mathrm{H}_{5}-\mathrm{N}=\mathrm{N}-\mathrm{C}_{6} \mathrm{H}_{4}-\mathrm{O}-\left(\mathrm{CH}_{2}\right)_{6}-\mathrm{S}-\mathrm{Au}$} \\
\hline $\mathrm{S} 2 p_{3 / 2}$ & $\mathrm{C}=\mathrm{C}$ & $\mathrm{C}-\mathrm{N}$ & $\mathrm{C}-\mathrm{O}$ & \multicolumn{5}{|l|}{ shake-up } \\
\hline $162.0(0.56)$ & 284.45 & - & 285.9 & \multicolumn{5}{|l|}{291.5} \\
\hline TF-Az6 & \multicolumn{8}{|c|}{$\mathrm{CF}_{3}-\mathrm{C}_{6} \mathrm{H}_{4}-\mathrm{N}=\mathrm{N}-\mathrm{C}_{6} \mathrm{H}_{4}-\mathrm{O}-\left(\mathrm{CH}_{2}\right)_{6}-\mathrm{S}-\mathrm{Au}$} \\
\hline $\mathrm{S} 2 p_{3 / 2}$ & $\mathrm{C}=\mathrm{C}$ & $\mathrm{C}-\mathrm{N} / \mathrm{C}-\mathrm{CF}_{3}$ & $\mathrm{C}-\mathrm{O}$ & shake-up & $\mathrm{C}-\mathrm{F}_{2}$ & $\mathrm{C}-\mathrm{F}_{3}$ & $\mathrm{~N} 1 s$ & N $1 s$ shake-up \\
\hline $162.0(0.57)$ & $284.5(0.89)$ & $285.1(0.78)$ & $286.0(1.25)$ & - & $289.9(1.1)$ & $292.2(0.95)$ & $399.6(1.2) / 399.8(1.0)$ & $401.7 / 403.1$ \\
\hline
\end{tabular}

i.e., for SAMs with even alkane chain-length $n$ [21]. Interestingly, the intensity of this redshifted component increased upon annealing, whereby the $\mathrm{BP} n$ SAMs underwent a phase transition between two structures with presumably different adsorption sites [21]. For well-prepared Az6 SAMs, we do not observe blueshifted components attributed to disulfides or oxidation products. They occur, however, in addition to atomic sulphur after long-term X-ray exposure.

The full width at half maximum (FWHM) of the $\mathrm{S} 2 p$ components amounts to $0.52 \mathrm{eV}$ for $\mathrm{C} 6$ and shows a slightly larger value of $0.56-0.57 \mathrm{eV}$ for Az6 and TF-Az6. The latter is also larger than the FWHM of $0.50 \mathrm{eV}$ reported for highly ordered BP $n$ SAMs on gold [20, 21]. Moreover, for Az6 and TF-Az6, the Au 4f $f_{7 / 2}$ level exhibits an FWHM of $0.62 \mathrm{eV}$ (not shown) as compared to $0.40-0.48 \mathrm{eV}$ for $\mathrm{BP} n, n=1-4$ [20]. The broadening of XPS lines is usually attributed to inhomogeneities in the film and may be caused by slightly different sulphur adsorption sites. This indicates that the Az6 and TF-Az6 SAMs show some disorder. Whether these lateral inhomogeneities are an equilibrium property of the SAMs or are determined by the adsorption kinetics needs to be clarified by further annealing experiments and immersion at elevated temperature.

Turning to the C $1 s$ XPS data in Fig. 3, a comparison of the same three different thiol compounds (C6, Az6, and TFAz6) reveals several chemically shifted emission lines. The TF-Az6 spectrum has been recorded for $\Delta R=0.1 \mathrm{eV}$, while the Az6 and C6 XPS measurements had only a moderate energy resolution of $0.6 \mathrm{eV}$. Therefore, we concentrate on a comparison of the binding energies and only discuss the linewidth for the TF-Az6 compound.

The binding energy of $284.6 \mathrm{eV}$ for the main component of the C6 alkanethiol is consistent with the values reported for short alkane chain-lengths [25]. The additional $\mathrm{C} 1 s$ component, shifted by $0.8 \mathrm{eV}$ to higher binding energies, amounts to $14 \%$ of the main-line intensity. Following a

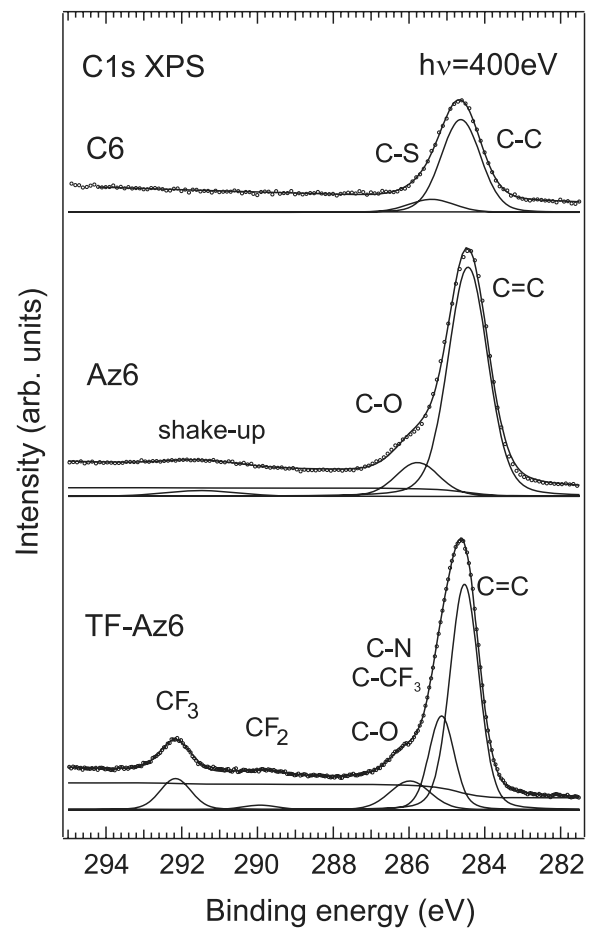

Fig. $3 \mathrm{XP}$ spectra of the $\mathrm{C} 1 s$ core level region of C6, Az6, and TF-Az6 on gold recorded at a photon energy of $400 \mathrm{eV}$. C6 and Az6 spectra have a moderate resolution of $\Delta R=0.6 \mathrm{eV}$, and TF-Az6 is a high resolution XP spectrum with $\Delta R \leq 0.1 \mathrm{eV}$. The spectra are decomposed into least square fits of Voigt profiles and a Shirley background [12]

recent study of non-substituted thioaromatic compounds, we attribute this second component to the sulphur-bound carbon atom [26]. This assignment is corroborated by our highresolution XPS measurements of $\mathrm{C} 3$ which exhibit a shift of the main line to $284.0 \mathrm{eV}$ and an increased intensity of the chemically shifted C $1 s$ component [27]. The former also confirms the general trend that for alkanethiols adsorbed on 
gold, the $\mathrm{C} 1 s$ binding energy shifts to higher values with increasing chain length $[25,28]$. This shift has been attributed to a gradual decrease of charge transfer screening with increasing length $n$, i.e., an increasing distance of the carbon atoms from the gold substrate [28].

The main line of the Az6 compound has a binding energy of $284.45 \mathrm{eV}$, which is $0.15 \mathrm{eV}$ larger than the binding energy reported for BP6 recorded at a photon energy of $350 \mathrm{eV}$ [21]. For photoelectrons with around $100 \mathrm{eV}$ kinetic energy, the attenuation length in alkanethiol SAMs amounts to about $5.4 \AA$ [29]. Thus, for the photon energy of $400 \mathrm{eV}$, the $\mathrm{C} 1 \mathrm{~s}$ XPS data are not quantitative, and the $\mathrm{C} 1 s$ signal of both the Az6 and BP6 compounds will be dominated by the carbon atoms of the aromatic moieties. Accordingly, the small shift of the $\mathrm{C} 1 s$ main line to higher binding energies for the photo-switch as compared to the biphenyl is likely caused by the azo group. In addition to the peak shift, we observe a pronounced shoulder to the left of the main line, i.e., at higher binding energies. Decomposing the $\mathrm{C} 1 s$ main line into two Voigt profiles yields a second component with a binding energy of $285.9 \mathrm{eV}$. We assign this component to the two carbon atoms of the phenoxy group in line with the shifts typically observed for carbon-oxygen single bonds [30]. The broad peak centered at $291.5 \mathrm{eV}$ is attributed to shake-up excitations in the $\pi$ and $\pi^{*}$ manifold accompanying the core ionization in the phenyl groups [30-32].

As to the high resolution $\mathrm{C} 1 s \mathrm{XP}$ spectrum of the TFAz6 SAM $(\Delta R=0.1 \mathrm{eV})$, the main XPS peak and its shoulder at high binding energies cannot be satisfactorily described by only two Voigt profiles. The necessary third component is attributed to the $\mathrm{C}-\mathrm{N}$ induced peak shift. Decomposition of the spectrum into three Voigt profiles results in binding energies of 284.5, 285.1, and $286.0 \mathrm{eV}$ assigned to the carbon atoms in the $2,3,5,6$ and $2^{\prime}, 3^{\prime}, 5^{\prime}, 6^{\prime}$ positions of the azobenzene moiety (Fig. 1), the carbon atoms 1 and $1^{\prime}$ at the azo group, and the $\mathrm{C} 1 s$ core-levels 4 and 8 of the phenoxy group as well as the $4^{\prime}$ carbon atom at the trifluoromethyl tailgroup. Compared to Az6, the main line is on average shifted to higher binding energies which is expected considering the charge pulling character of the $\mathrm{CF}_{3}$ tailgroup. The most obvious difference between the TF-Az6 and Az6 spectra is the strongly shifted C $1 s$ component of the trifluoromethyl group at a binding energy of $292.2 \mathrm{eV}$ in line with HR-XPS data of modified polyimides [33].

The FWHM of $0.95 \mathrm{eV}$ of the trifluoromethyl group is attributed to vibrational broadening [34]. In XAS this group can in principle be used as an independent marker to determine the tilt of the molecules, but, as always with fluorinated hydrocarbons, great care has to be taken with respect to X-ray induced beam damage. Finally we note that we do not need to introduce the shake-up intensity seen for Az6 at $291.5 \mathrm{eV}$ to describe the spectra of TF-Az6. The tiny peak at $289.9 \mathrm{eV}$ is assigned to $\mathrm{CF}_{2}$ as discussed in the following.

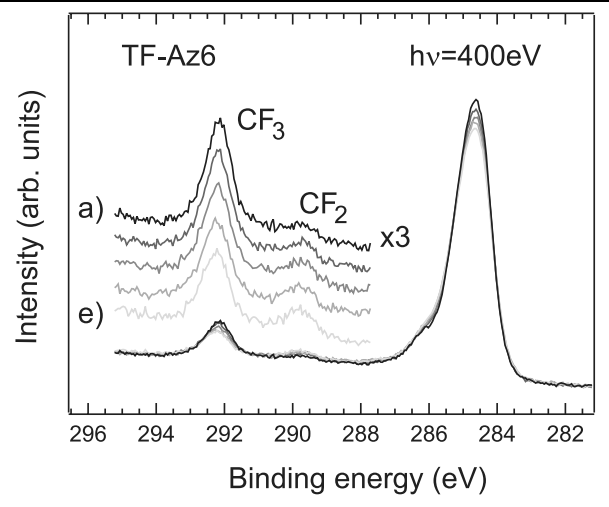

Fig. 4 High-resolution $\mathrm{C} 1 s \mathrm{XP}$ spectrum of TF-Az6 recorded at a photon energy of $400 \mathrm{eV}$. Each measurement corresponds to an X-ray exposure of $2.3 \times 10^{15}$ photons $/ \mathrm{cm}^{2}$. With increasing exposure from a) to e), the $\mathrm{CF}_{3}$ intensity decreases, and a new peak grows, which is assigned to $\mathrm{CF}_{2}$ group

\subsection{X-ray induced beam damage}

Radiation-induced damage is a severe problem in X-ray spectroscopy of organic layers [22, 35]. In general it is believed that secondary electrons are responsible for beam damage, but also resonant X-ray core excitations are effective in bond breaking [36]. For aliphatic SAMs, cooling suppresses the transport of heavy fragments, but already an exposure of $5 \times 10^{16}$ photons $/ \mathrm{cm}^{2}$ leads to significant formation of carbon double-bonds and of atomic sulphur $[23,24]$. Combined aliphatic-aromatic SAMs are less sensitive to electron bombardment, and X-ray beam damage is believed to be reduced [37]. For X-ray spectroscopy, we use a typical intensity of $4 \times 10^{13}$ photons $/ \mathrm{cm}^{2} \mathrm{~s}$. The power is determined by a GaAsP-Schottky-diode $[38,39]$ and the beam size by a phosphor mounted in the plane of the sample holder. For a typical XPS measurement and a $30 \mathrm{eV}$ XAS scan, this corresponds to an exposure of $10^{16}$ photons $/ \mathrm{cm}^{2}$. This requires that for XAS the beam is blocked by a fast shutter while monochromator and undulator are moving. As seen from Fig. 1 in [23], such a fluence is even tolerable for aliphatic SAMs, and we do not observe changes in the sulphur XP spectrum.

However, for fluorinated hydrocarbons, beam damage is by far worse. This is illustrated by the series of $\mathrm{C} 1 \mathrm{~s}$ highresolution XP spectra of TF-Az6 in Fig. $4(\Delta R=0.1 \mathrm{eV})$, where each consecutive spectrum corresponds to an exposure of $2.3 \times 10^{15}$ photons $/ \mathrm{cm}^{2}$. As already discussed, the peak at $292.2 \mathrm{eV}$ is assigned to the $\mathrm{CF}_{3}$ marker group. The $\mathrm{CF}_{3}$-related intensity strongly decreases under continuing $\mathrm{X}$-ray exposure, and a second peak simultaneously grows at a binding energy of $289.9 \mathrm{eV}$ attributed to the $\mathrm{CF}_{2}$ radical. Already after an exposure of $10^{16}$ photons $/ \mathrm{cm}^{2}$, the $\mathrm{CF}_{3}$ intensity has dropped by $22 \%$, and we observe an increase of the $\mathrm{CF}_{2}$ component. For comparison, the intensity of the main peak decreased for the same exposure by 


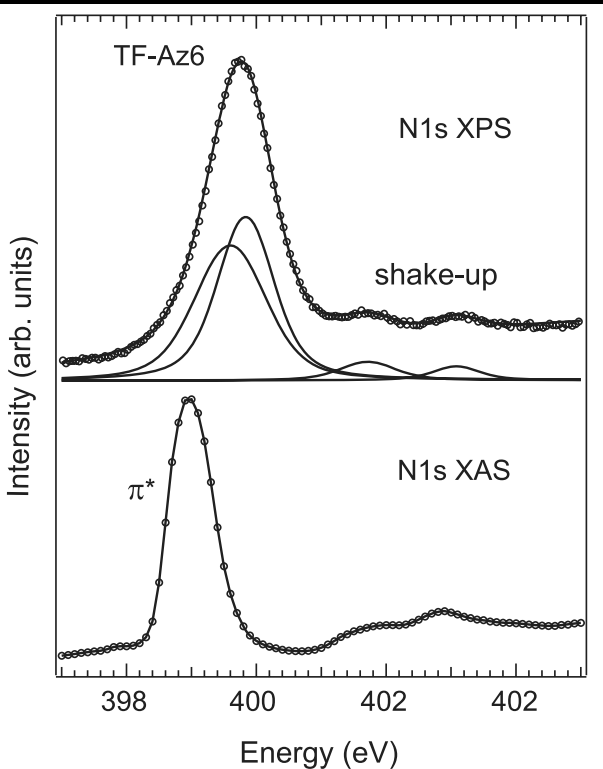

Fig. 5 High-resolution XP spectrum of the N $1 s$ region of TF-Az6 and the corresponding XA spectrum of the $\mathrm{N} 1 s$ to $\pi^{*}$ transition. The abscissae refers to the ascending binding energy in case of XPS and to the photon energy in case of XAS. The XPS data were recorded at a photon energy of $500 \mathrm{eV}$ with a resolution of $\Delta R=0.2 \mathrm{eV}$

only $3 \%$, while the FWHM of $1.1 \mathrm{eV}$ broadens by $0.1 \mathrm{eV}$. This corroborates the interpretation that the aromatic moiety is rather robust against radiation-induced damage, while the trifluromethyl group shows significant bond breaking already at low X-ray exposure.

\subsection{Shake-up transitions}

We have already assigned a small broad peak in the C $1 s$ XP spectrum of Az6 to a shake-up satellite centered at an energy of $7.7 \mathrm{eV}$ above the main line, cf. Fig. 2. In comparison, inspecting the N $1 s$ XP spectrum of TF-Az6 in the upper part of Fig. 5, we see weak shake-up satellites at significantly lower energies of about 2.1 and $3.5 \mathrm{eV}$ above the main line. Note that for reasons that we will discuss below, the energy scale in Fig. 5 is inverted as compared to Fig. 2 so that shake-up peaks appear to the right of the main line. For the free benzene and phenol $\left(\mathrm{C}_{6} \mathrm{H}_{5} \mathrm{OH}\right)$ molecule, several monopole shake-up transitions are found in the energy range between 5 and $11 \mathrm{eV}$ [30]. These satellites are attributed to $\pi-\pi^{*}$ transitions within the electronic manifold of the phenyl ring. We note that for the free benzene molecule, the ground-state $D_{6 h}$ symmetry is reduced to $C_{2 v}$ upon creation of a core hole. This leads to a splitting of the initially degenerate $\pi$ and $\pi^{*}$ levels and thus explains the spread of individual shake-up transitions over an energy range of $6 \mathrm{eV}$. In the phenol or azobenzene, this splitting of the $\pi$ system occurs already in the ground state. Nevertheless, at first view the shake-up regions of benzene and phenol XP spectra dif-

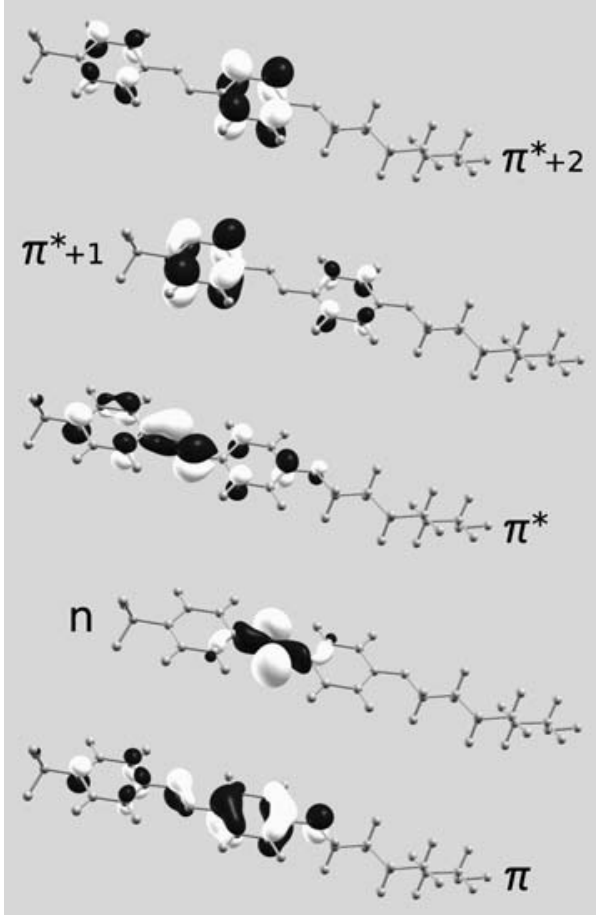

Fig. 6 Isodensity-contour plots of $\pi$ HOMO-1, $n$ HOMO, $\pi^{*}$ LUMO, and the nearly degenerate $\pi^{*}+1$ and $\pi^{*}+2$ LUMO +1 of the free TF-Az6 molecule

fer little, and also for simple polymers, shake-up transitions are found in the same energy range $[31,33]$.

In azobenzene the $\pi$ and $\pi^{*}$ levels correspond to the HOMO - 1 and LUMO, while the lone-pair orbital $n$ of the azo group is the HOMO (Highest Occupied Molecular Orbital). Calculated isodensity contour plots of these orbitals as well as of the LUMO $+1 \pi$ levels are depicted in Fig. 6. Optical spectroscopy in ethanolic solution yields absorption bands at about 2.8 and $3.6 \mathrm{eV}$ assigned to the $n-\pi^{*}$ and $\pi-\pi^{*}$ transitions in line with our time-dependent DFT calculations of the free molecule. Our angle-resolved valenceband photoemission of TF-Az6 reveals binding energies of 2.8 and $4.0 \mathrm{eV}$ for $n$ and $\pi$, respectively. Based on these energies of valence orbitals and photoemission final states, we assign the two satellites in the $\mathrm{N} 1 s \mathrm{XP}$ spectrum at 2.1 and $3.5 \mathrm{eV}$ above the mainline to $n-\pi^{*}$ and $\pi-\pi^{*}$ shakeups. The difference of $1.4 \mathrm{eV}$ between the two satellites is slightly larger than the separation of $1.2 \mathrm{eV}$ in photoemission and significantly larger than the distance of $0.8 \mathrm{eV}$ of the optical absorption bands. However, energies of shakeup satellites are strongly influenced by final state effects, and transition energies include the interaction of the excited electron-hole pair with the core hole.

The shake-up satellites in the $\mathrm{C} 1 s \mathrm{XP}$ spectrum are centered at $7.2 \mathrm{eV}$ above the main line. They cannot reflect excitations to the $\pi^{*}$ LUMO and must therefore involve transitions to the higher lying unoccupied $\pi$ orbitals. Inspecting the orbital contour-plots in Fig. 6, we see that the $\pi^{*}$ orbital 
is distributed over the whole azobenzene moiety but with the highest probability density at the nitrogen atoms. The $n$ orbital concentrates at the azo group, while the $\pi$ orbital is mainly located at the $\alpha$-phenyl with some contribution also at the azo group. The two $\pi$ orbitals denoted as $\pi^{*}+1$ and $\pi^{*}+2$ display together a rather symmetric probability density located at the phenyl rings of the azobenzene entity. In Az6 the computed charge distribution of these two orbitals is even somewhat more symmetrically distributed among the phenyl rings, since the charge-pulling $\mathrm{CF}_{3}$ group is replaced by a hydrogen atom. These orbitals are therefore good candidates as shake-up final states upon $\mathrm{C} 1 s$ excitation since the probability density is mainly located at the phenyl ring. The latter orbitals are nearly degenerated and found at $1.7 \mathrm{eV}$ above the LUMO. They show the same energy shift in XAS spectroscopy at the $\mathrm{C} 1 s$ absorption edge. Therefore, we expect shake-up transitions into these orbitals starting at about $5.3 \mathrm{eV}$ above the main line. This explains the different positions of the XPS satellites in the carbon and nitrogen spectra of the azobenzene moiety.

For the free benzene molecule, the total intensity of the $\pi-\pi^{*}$ shake-up region amounts to about $15 \%$ of the main line and is little affected by the hydroxyl group in the case of the phenol molecule [30]. In the Az6 SAM the intensity of the shake-up satellites is reduced to $4 \%$. This is similar to the findings for aromatic polymers $[33,40]$ or benzene weakly chemisorbed on $\mathrm{Cu}(110)$ [32]. From the autoionization studies of Feulner and coworkers [41] we conclude that the alkane chain decouples the azobenzene chromophore from the surface rather effectively, and we would expect a high intensity of the satellites. The observed weak contribution of distinct shake-up transitions to the XP spectrum thus suggests that intermolecular interactions in the SAM are strong and lead to a quenching of intramolecular excitations.

\subsection{Comparison to the near edge X-ray absorption fine structure}

Polarization-dependent XAS was used to determine the orientation of the azobenzene moieties in the SAM. We observe a molecular orientation similar to BP $n$ SAMs with odd numbered chain length [37] and will give a detailed analysis elsewhere [27]. Here we concentrate on the relation between core hole to bound-state excitation in XAS and core ionization in XPS. For this purpose, high-resolution XP and XA spectra of TF-Az6 are plotted on a common energy scale in Fig. 5 and Fig. 7 for the nitrogen and the carbon coreexcitations, respectively. For the XP spectrum, the abscissae refers to the binding energy, while for XAS, the abscissae refers to the photon energy.

The $\mathrm{N} 1 s$ to $\pi^{*}$ transition is a strong peak with a FWHM of $1.0 \mathrm{eV}$. The $\pi^{*}$ resonance at the carbon edge is broader

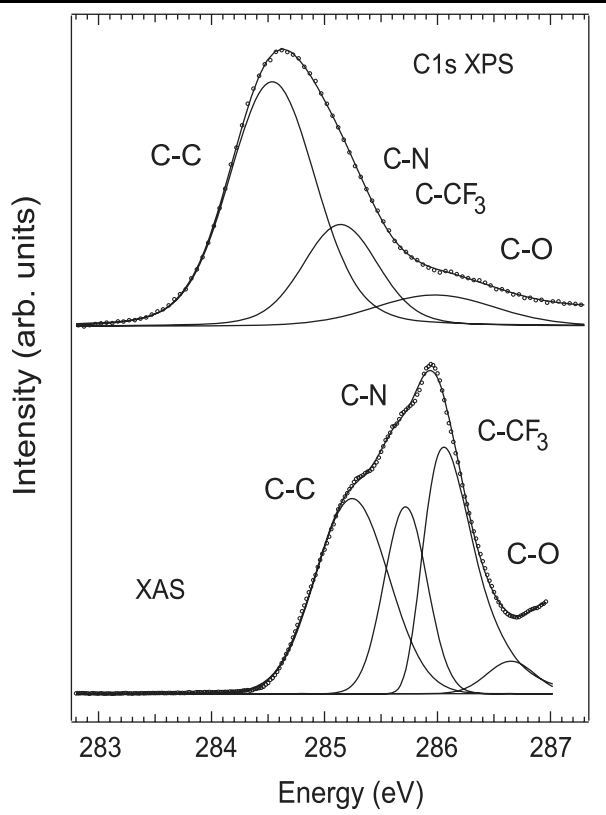

Fig. 7 High-resolution XP spectrum of the $\mathrm{C} 1 s$ region of TF-Az6 and the corresponding XA spectrum of the $\mathrm{C} 1 s$ to $\pi^{*}$ transition. The abscissae refers to the ascending binding energy in case of XPS and to the photon energy in case of XAS. The XPS data were recorded at a photon energy of $400 \mathrm{eV}$ with a resolution of $\Delta R=0.1 \mathrm{eV}$. The XA spectrum is decomposed into four components assigned to the different carbon atoms of the azobenzene moiety of TF-Az6

and exhibits a step-like fine structure with at least three components. As seen from Fig. 6, the LUMO spreads over the azobenzene moiety with high probability density at the nitrogen atoms. This is in line with the salient intensity of the $\mathrm{N} 1 s$ resonance and the splitting of the $\mathrm{C} 1 s$ to $\pi^{*}$ transition. The latter is attributed to different chemical surroundings of the carbon atoms. A tentative assignment based only on calculated initial-state energy differences is given in Fig. 7 [27].

Most noticeably, the relative energies $\Delta E_{r e s}$ of neutral excitations and core ionization differ for nitrogen and carbon. While the N $1 s$ XPS peak lies above the $\pi^{*}$-resonance at $\Delta E_{\text {res }}=-0.7 \mathrm{eV}$, the $\mathrm{C} 1 \mathrm{~s}$ XPS peak is centered at about $\Delta E_{\text {res }}=0.6 \mathrm{eV}$ below the corresponding $\mathrm{C} 1 s$ to $\pi^{*}$ transition. We have referenced the XPS binding energies to the Au $4 f$ line and thereby to the Fermi level of the substrate. In other words, the XPS binding energy corresponds to the energy required to excite an electron from the core hole to the Fermi level, since the energy of one extra electron at the Fermi level is insignificant. If we allowed for charge transport between the gold substrate and the azobenzene chromophore, the current upon $\pi^{*}$-excitation would depend on the type of core hole. In the presence of a $\mathrm{C} 1 s$ core hole charge transfer from the LUMO of the chromophore to the substrate is energetically allowed, while it is forbidden in the presence of a N $1 s$ core hole. Here for energetic reasons electrons would flow from the substrate to the chromophore upon ionizing the $1 s$ level. However, we cannot 
have metallic screening, since in this case the XPS binding energy is expected to be lower than or equal to the XAS transition energy. Thus we argue that we have weak polarization screening of the core-excited states. This case was, e.g., observed for $\mathrm{N}_{2}$ on graphite, where the Auger spectrum following core ionization showed decay from both an ionic and a neutral intermediate state [42]. The interpretation is also in line with recent results by Feulner and coworkers, who proved that for alkanethiol SAMs with nitrile tailgroup, charge transfer in the $\mathrm{N} 1 s \pi^{*}$-resonance depends strongly on the length of the aliphatic chain [41]. For a chain length of $n=16$, no charge transfer occurred, and the N $1 s$ XPS line was found at $\Delta E_{\text {res }}=-0.2 \mathrm{eV}$, i.e., above the $\pi^{*}$-resonance transition. For a chain length of $n=2$, the order of coreneutral and ionic excitation was reversed $\Delta E_{\text {res }}=+1.2 \mathrm{eV}$, and charge transfer from the nitrile tailgroup to the gold surface was observed. Extrapolating the observed chargetransfer time of 13 fs to a chain length of $n=6$, we obtain picoseconds. As expected, on the femtosecond time scale of core-hole decay, charge transfer to the substrate is negligible. However, in first autoionization experiments we observe non-resonant contributions in the decay of both the nitrogen and carbon core-hole. Thus charge transfer on the femtosecond time scale defined by the lifetime of the core hole must occur among the azobenzene moieties which may, however, depend on the degree of localization of the core hole. We therefore conclude that we encounter a situation where charge transfer is not dominated by the substrate but by the azobenzene moieties in the SAM. This requires a densely packed SAM where the orbitals tend to form electronic bands. The interaction between the core hole and the surrounding will to some extent localize the charge distribution in the core-excited state. This may differ for the azo group as compared to the $\pi$-system of the phenyl rings. The push-pull system formed by the oxygen bridge and the trifluoromethyl tailgroup may further modify the intermolecular interactions, since it leads to a less symmetric charge distribution already in the ground state (cf. the $\pi$ orbital in Fig. 6). For TF-Az6, we do observe shake-up excitations upon N $1 s$ core ionization but not in the C $1 s$ XP spectrum. Here we observe XPS satellites only for the Az6 SAM. Again we attribute the different shake-up intensities for nitrogen and carbon to differences in screening of the respective core hole.

\subsection{Final-state screening}

Changes in the XPS binding energy of one molecule are due to differences in the total energy of the molecule in the final, core ionized state [43]. Using the equivalent core approximation, we can replace the core ionized atom by its $Z+1$ element. This way we expect, e.g., very small shifts between the two nitrogen atoms, since replacing either of the two nitrogen atoms by an oxygen atom the local molecular structure differs first for the 4 and $4^{\prime}$ positions. However, when

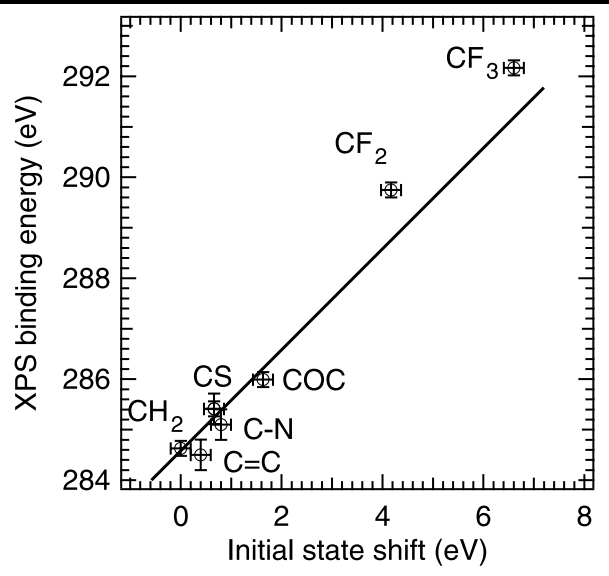

Fig. 8 Calculated initial-state shifts versus C $1 s$ XPS binding energy as derived from Fig. 3

comparing the carbon atoms of the azobenzene moiety, we cannot directly motivate the total binding energy of the final state and thereby predict chemical shifts. Therefore we have used a very rough approach to estimate chemical shifts on the basis of calculated initial-state shifts of single-particle energies [19]. Since neither screening nor correlation effects are accounted for, we have to assume that relaxation effects are of comparable size for all the atoms of the molecule. That this is a rather reasonable approach for the carbon atoms of the aliphatic-aromatic SAMs is demonstrated by a comparison of the measured $\mathrm{C} 1 s$ binding energies and calculated initial-state shifts in Fig. 8. Data points related to the $\mathrm{C} 6$ alkane, the C-S-gold bond, the azo and the phenoxy group string together along a straight line with slope one. The $\mathrm{CF}_{2}$ and $\mathrm{CF}_{3}$ tailgroups show a larger binding energy, which is attributed to enhanced screening in the final state. Furthermore, the difference between the headgroup which binds to the gold substrate and the tailgroup which defines the SAM-vacuum interface is comparable to polymer multilayers [33]. In the SAMs headgroup and chromophore with tailgroup are separated by the alkane chain. As already mentioned, on the femtosecond time scale of core excitation and decay, charge transfer across this linker is very slow [41]. This again indicates that screening of the $\mathrm{C} 1 s$ core holes in the azobenzene chromophore must be mediated by sizeable intra- and intermolecular screening.

\section{Conclusions}

Azobenzene-alkanethiols form SAMs of high molecular order on the gold surface. The 4-trifluoro-methyl-azobenzene4 '-methyleneoxy-alkanethiols show the typical thiolate bond with a single adsorbed species. The comparison of XPS binding energies and $\pi^{*}$-excitation energies of the azobenzene chromophore suggests that screening of the N $1 s$ XPS 
final state is weak and preferentially mediated by polarization. In contrast, the $\mathrm{C} 1 s$ XPS final state has a lower energy than the neutral $1 s^{-1} \pi^{+1}$ excitation. Thus the ionic state must involve sizeable intra- and intermolecular screening. This is substantiated by a comparison of measured $\mathrm{C} 1 \mathrm{~s}$ binding energies and calculated initial-state chemical shifts. We propose that the strong intermolecular coupling also leads to a rapid decay of optical excitations and could therefore explain the low photo-switching activity observed for azobenzene in densely packed aromatic-aliphatic SAMs on gold [44].

Acknowledgement Support by the Deutsche Forschungsgemeinschaft through Sfb 658-Elementary Processes in Molecular Switches at Surfaces-is gratefully acknowledged. We thank Peter Feulner for his lasting technical support and again him and Wilfried Wurth for very valuable discussions.

\section{References}

1. T. Nägele, R. Hoche, W. Zinth, J. Wachtveitl, Chem. Phys. Lett. 272, 489 (1997)

2. T. Fujino, S.Y. Arzhantsev, T. Tahara, J. Phys. Chem. A 105, 8123 (2001)

3. M.J. Comstock et al., Phys. Rev. Lett. 99, 038301 (2007)

4. S. Hagen, F. Leyssner, D. Nandi, M. Wolf, P. Tegeder, Chem. Phys. Lett. 444, 85 (2007)

5. M. Zharnikov, M. Grunze, J. Phys.: Condens. Matter 13, 11333 (2001)

6. P. Cyganik, M. Buck, J.D.E.T. Wilton-Ely, C. Wöll, J. Phys. Chem. B 109, $10902(2005)$

7. S. Frey, H.-T. Rong, K. Heister, Y.-J. Yang, M. Buck, M. Zharnikov, Langmuir 18, 3142 (2002)

8. P. Kowalczyk, Appl. Surf. Sci. 253, 4036 (2007)

9. D. Graham, S. Dingman, Mater. Matters 1, 18 (2006)

10. C. Jung, F. Eggenstein, S. Hartlaub, R. Follath, J.S. Schmidt, F. Senf, M. Weiss, T. Zeschke, W. Gudat, Nucl. Instr. Meth. A 467, $485(2001)$

11. M.P. Seah, Surf. Interface Anal. 31, 721 (2001)

12. D.A. Shirley, Phys. Rev. B 5, 4709 (1972)

13. M.J. Frisch, G.W. Trucks, H.B. Schlegel, G.E. Scuseria, M.A. Robb, J.R. Cheeseman, J.A. Montgomery Jr., T. Vreven, K.N. Kudin, J.C. Burant, J.M. Millam, S.S. Iyengar, J. Tomasi, V. Barone, B. Mennucci, M. Cossi, G. Scalmani, N. Rega, G.A. Petersson, H. Nakatsuji, M. Hada, M. Ehara, K. Toyota, R. Fukuda, J. Hasegawa, M. Ishida, T. Nakajima, Y. Honda, O. Kitao, H. Nakai, M. Klene, X. Li, J.E. Knox, H.P. Hratchian, J.B. Cross, C. Adamo, J. Jaramillo, R. Gomperts, R.E. Stratmann, O. Yazyev, A.J. Austin, R. Cammi, C. Pomelli, J.W. Ochterski, P.Y. Ayala, K. Morokuma, G.A. Voth, P. Salvador, J.J. Dannenberg, V.G. Zakrzewski, S. Dapprich, A.D. Daniels, M.C. Strain, O. Farkas, D.K. Malick, A.D. Rabuck, K. Raghavachari, J.B. Foresman, J.V. Ortiz, Q. Cui, A.G. Baboul, S. Clifford, J. Cioslowski, B.B. Stefanov, G. Liu, A. Liashenko, P. Piskorz, I. Komaromi, R.L. Martin, D.J. Fox, T. Keith, M.A. Al-Laham, C.Y. Peng, A. Nanayakkara, M. Challacombe, P.M.W. Gill, B. Johnson, W. Chen, M.W. Wong, C. Gonzalez, J.A. Pople, Gaussian 03, Revision B. 05 (Gaussian Inc., Pittsburgh, 2003)
14. P.J. Stephens, F.J. Devlin, C.F. Chabalowski, M.J. Frisch, J. Phys. Chem. 98, 11623 (1994)

15. M.E. Casida, C. Jamorski, K.C. Casida, D.R. Salahub, J. Chem. Phys. 108, 4439 (1998)

16. R. Bauernschmitt, R. Ahlrichs, Chem. Phys. Lett. 256, 454 (1996)

17. A. Schäfer, C. Huber, R. Ahlrichs, J. Chem. Phys. 100, 5829 (1994)

18. W. Kutzelnigg, U. Fleischer, M. Schindler, in NMR-Basic Principles and Progress, vol. 23 (Springer, Heidelberg, 1990), p. 165

19. S. Lizzit et al., Phys. Rev. B 63, 205419 (2001)

20. K. Heister, H.-T. Rong, M. Buck, M. Zharnikov, M. Grunze, L.S.O. Johansson, J. Phys. Chem. B 105, 6888 (2001)

21. P. Cyganik, M. Buck, T. Strunskus, A. Shaporenko, J.D.E.T. Wilton-Ely, M. Zharnikov, C. Wöll, J. Am. Chem. Soc. 128, 13868 (2006)

22. K. Heister, M. Zharnikov, M. Grunze, L.S.O. Johansson, A. U1man, Langmuir 17, 8 (2001)

23. P. Feulner, T. Niedermeyer, K. Eberle, R. Schneider, D. Menzel, A. Baumer, E. Schmich, S. Shaporenko, Y. Tai, M. Zharnikov, Phys. Rev. Lett. 93, 178302 (2004)

24. P. Feulner, T. Niedermayer, K. Eberle, R. Schneider, D. Menzel, A. Baumer, E. Schmich, A. Shaporenko, Y. Tai, M. Zharnikov, Surf. Sci. 593, 252 (2005)

25. H. Biebuyck, C. Bain, G. Whitesides, Langmuir 10, 1825 (1994)

26. A. Shaporenko, A. Terfort, M. Grunze, M. Zharnikov, J. Electron Spectrosc. Relat. Phenom. 151, 45 (2006)

27. R. Schmidt, E. McNellis, D. Brete, T. Gießel, W. Freyer, C. Gahl, K. Reuter, M. Weinelt (2008 in preparation)

28. K. Heister, L.S.O. Johansson, M. Grunze, M. Zharnikov, Surf. Sci. 529, $36(2003)$

29. C.L.A. Larmont, J. Wilkes, Langmuir 15, 2037 (1999)

30. D. Nordfors, A. Nilsson, N. Mårtensson, S. Svensson, U. Gelius, S. Lunell, J. Chem. Phys. 88, 2630 (1988)

31. D.T. Clark, D.B. Adams, A. Dilks, J. Peeling, H.R. Thomas, J. Electron Spectrosc. Relat. Phenom. 8, 51 (1976)

32. M. Weinelt, N. Wassdahl, T. Wiell, O. Karis, J. Hasselström, P. Bennich, A. Nilsson, J. Stöhr, M. Samant, Phys. Rev. B 58, 7351 (1998)

33. J.E. Fulghum, J. Electron Spectrosc. Relat. Phenom. 100, 331 (1999)

34. H.-P. Steinrück, T. Fuhrmann, C. Papp, B. Tränkenschuh, R. Denecke, J. Chem. Phys. 125, 204706 (2006)

35. K. Heister, S. Frey, A. Ulman, M. Grunze, M. Zharnikov, Langmuir 20, 1222 (2004)

36. D. Menzel, P. Feulner, J. Phys. Cond. Matt. 13, 11249 (2001)

37. H.-T. Rong, S. Frey, Y.-J. Yang, M. Zharnikov, M. Buck, M. Wühn, C. Wöll, G. Helmchen, Langmuir 17, 1582 (2001)

38. M. Krumrey, E. Tegeler, J. Barth, M. Krisch, F. Schafers, R. Wolf, Appl. Optics 27, 4336 (1988)

39. G. Reichardt, Berliner Elektronenspeicherring-Gesellschaft für Synchrotronstrahlung $\mathrm{GmbH}$, private communication

40. J. Gardella Jr., S. Ferguson, R. Chin, Appl. Spectrosc. 40, 224 (1986)

41. S. Neppl, U. Bauer, D. Menzel, P. Feulner, A. Shaporenko, M. Zharnikov, P. Kao, D. Allara, Chem. Phys. Lett. 447, 227 (2007)

42. O. Björneholm, A. Nilsson, A. Sandell, B. Hernnäs, N. Mårtensson, Phys. Rev. Lett. 68, 1892 (1992)

43. N. Mårtensson, A. Nilsson, J. Electron Spectrosc. Relat. Phenom. 75, 209 (1995)

44. R. Wang, T. Iyoda, L. Jiang, D.A. Tryk, K. Hashimoto, A. Fujishima, J. Electroanal. Chem. 438, 213 (1997) 